DOI:

10.1016/j.jjms.2016.01.009

\title{
Relative ion intensities of maltooligosaccharide ethers in electrospray ionization ion trap mass spectrometry: A quantitative evaluation
}

Sheetal Gangula ${ }^{1}$, Manfred Nimtz ${ }^{2}$, Petra Mischnick ${ }^{l *}$

${ }^{1}$ Technische Universität Braunschweig, Institute of Food Chemistry, Schleinitzstr. 20, D-38106 Braunschweig, Germany

\section{${ }^{2}$ Helmholtz-Centre for Infection Research, Inhoffenstraße 7, D-38124 Braunschweig, Germany}

\section{ABSTRACT}

Electrospray ionization coupled with mass spectrometry (ESI-MS) is an important analytical technique for the analysis of polysaccharide derivatives. It can be used to analyze substitution patterns in mixtures of oligomers to recognize heterogeneities, bimodality or blocky sequences. Quantitative analysis is hampered, however, by the fact that compounds of different chemistry and molar mass usually show different ionization efficiencies. In order to study the parameters of influence and to understand whether relative ion intensities in MS follow certain patterns. We analyzed border-eases fully $O$-methylated and fully $O$-perdeuteriomethylated oligosaccharides as two extreme cases of possible mixed $O$-Me- $/ O-\mathrm{Me}-d_{3}$ by ESI-MS under defined conditions. Binary and complex mixtures of maltooligosaccharide ethers from DP 1 to 7 by were prepared from partially hydrolyzed per- $O$-methyl$\beta$-cyclodextrin (Me- $\beta-\mathrm{CD})$ and per-O-deuteromethyl- $\beta$-cyclodextrin $\left(\mathrm{Me}-d_{3}-\beta-\mathrm{CD}\right)$. Reference data for their real compositions were obtained after reductive amination by HPLC-UV. Within the applicable concentration range and with properly adjusted instrumental parameters, relative sensitivities $\left(\mathrm{Me}-d_{3} / \mathrm{Me}\right)$ in ESI-MS remained constant for a respective DP. With increasing DP, the relative sensitivity coefficients decreased from 1.01 for DP 2 to 0.78 for DP 6 for the binary mixtures and showed similar behavior for complex mixtures. The decrease in relative sensitivity coefficients for corresponding Me- $d_{3}$ and Me derivatives has been overcome by $m$-amino-benzoic acid labeling and ESI-MS in negative mode or by measuring the original mixtures in nano-ESI-MS. Thus, discrimination probably caused by differences in surface activity and electrophoretic mobility can be leveled by an appropriate tag or formation of smaller droplets as in nano-ESI.

*corresponding author: p.mischnick@tu-braunschweig.de 


\section{INTRODUCTION}

Naturally occurring polysaccharides such as cellulose and starch, are modified by esterification or etherification of their free hydroxyl groups for various applications [1]. Such derivatives are employed in the construction area, for pharmaceuticals, food, cosmetics, and in the packaging industries, to name but a few applications. The physicochemical properties of these chemically modified biopolymers such as solubility, biodegradability, viscosity, or thermoreversible gelation, depend not only on the type of substituent and the average degree of substitution (DS), but also on the distribution in the glucosyl unit both along and over the macromolecule chains [2-4]. The molar ratios of the constituents varying in position and number of substituents are usually determined by NMR spectroscopy, GLC, CE or HPLC analysis of the depolymerized sample [5]. To gain information on the substitution pattern on the different structural levels, random degradation followed by mass spectrometric analysis of the DS profiles of sets of oligomers and comparison of these to a statistical model is applied [6-9]. To prevent discrimination during hydrolysis and subsequent MS analysis, methyl celluloses are permethylated with deuteromethyl iodide, resulting in chemically uniform polymers. MALDI-ToF-MS and ESI-IT-MS are applied as well, while the latter provides the advantage of easily being coupled with liquid chromatography (LC-ESI-IT-MS) and thus automation [10]. In the case of other MCs, for instance, $O$-ethyl- $O$-methyl glucans, this approach is hampered, however, by the fact that relative ion abundance in mass spectra does not necessarily represent the actual molar composition of the sample. Here, the compounds of lower polarity are detected with higher intensity, which causes distortion of MS profiles. $O$-Methyl- $O$-methyl- $d_{3}$ oligomers are chemically more similar and thus ESI-IT-MS data can be used for quantitative analysis. In monomer analysis regioisomeric $O$-methyl- $O$-methyl- $d_{3^{-}}$ glucose derivatives cannot be separated, though, while $O$-ethyl- $O$-methyl homologs can. Thus, it is of interest to extend the 'oligomer analysis' to chemically more different mixed ethers. As a prerequisite, the influence of the chemistry and molar mass (DP) on relative ion 
intensities in ESI-IT-MS needs to be investigated, starting with the well-known system $O$ $\mathrm{Me} / O-\mathrm{Me}-d_{3}$ as a reference. In a subsequent paper we will present the results for chemically more different glucooligosaccharide ethers.

Mechanism of formation of gas phase ions from solution in ESI is of interest for many researchers. This process is described in three major stages [11]: The sample solution is infused into a high electric field; a 'Taylor cone' is formed at the tip of the needle. Consequently, charged droplets are expelled $[12,13]$, and from these droplets gas phase ions are formed. Two main theories are widely accepted and have been experimentally investigated with respect to the mechanism of formation of gas phase ions from these charged droplets: the ion evaporation model (IEM) and the charge residue model (CRM) [14-16]. Solvated ions diffuse to and escape from the droplet surface. The ion yields of analyte molecules depend on their basicity or cation complexation ability (positive mode), polarity and surface activity of the analyte, its electrophoretic mobility and (de)solvation energy [17]. Therefore, it is plausible that even a small structural change can cause huge differences in relative ion intensities in ESI-MS. For quantifying the molar ratios of analytes from relative ion intensities, their dependence on sample composition and concentration must also be considered. Furthermore, with respect to instrumental parameters, flow rate [18] is also critical, because lower flow rates produce smaller droplets. Hence diffusion of analyte molecules to the surface of the droplet is affected and number of Coulomb explosions as well. Therefore, nano-ESI-MS [19] was applied for some selected samples. Finally, transmission of ions to the mass analyzer, in our case an ion trap, is affected by skimmer voltages and further parameters $[20]$.

To gain insight into the influence of all these parameters on relative ion intensities, fundamental studies of ESI-IT-MS with oligosaccharide derivatives are required. For the preparation of defined sample solutions, cyclodextrins rather than polysaccharides were 
chosen. $\beta$-Cyclodextrin was permethylated and perdeuteromethylated, respectively. Corresponding 2,3,6-tri- $O$-substituted maltooligosaccharides of DP 1-7 were obtained by partial hydrolysis and used for the preparation of mixed ethers. These were systematically studied by ESI-IT-MS. From our experience with mixed $O$-Me- $O-\mathrm{Me}-d_{3}$ oligosaccharides we expected close to equal ion intensities in the mass spectrum referred to equimolar concentration. Using this as a basis, we studied the behavior of uniformly $O$-methyl and corresponding $O$-methyl- $d_{3}$ maltooligosaccharides of DP 2-6 in ESI-IT-MS, which represent the two extreme cases in oligomer analysis of partial hydrolysates of perdeuteromethylated methyl glucans [10]. It is our long-term goal to establish whether the differences in ion yields of homolog series of oligosaccharide derivatives follow certain rules, which would allow correcting the data of a measurement of a mixture of unknown composition for quantification of molar ratio from relative ion intensities.

\section{EXPERIMENTAL}

\subsection{Reagents and materials}

All chemicals purchased were of highest purity and used without further purification. Iodomethane (MeI), iodomethane- $d_{3}\left(\mathrm{Me}-d_{3}-\mathrm{I}\right), m$-aminobenzoic acid ( $\left.m \mathrm{ABA}\right)$ and 2-picoline borane were purchased from Sigma Aldrich. Solvents used for ESI-MS were LC-MS grade and were purchased from Fluka.

\subsection{Synthesis}

Per- $O$-methylated- $\beta$-cyclodextrin $(\mathrm{Me}-\beta-\mathrm{CD})$ and per- $O$-deuteromethylated- $\beta$-cyclodextrin (Me- $\left.d_{3}-\beta-\mathrm{CD}\right)$ were synthesized according to Ciucanu and Kerek [21] in DMSO with $\mathrm{NaOH} / \mathrm{MeI}$ or MeI- $d_{3}$, respectively. Products were purified by re-crystallization from acetone. Completeness of alkylation was checked by the absence of $\mathrm{OH}$-absorption $>3000 \mathrm{~cm}^{-1}$ by ATR-IR spectroscopy. ${ }^{1}$ H-NMR spectra showed only a single doublet peak for $\mathrm{H}-1$ confirming the uniformity and were in agreement with the literature [22]. GLC analysis was 
performed after total hydrolysis, reduction and acetylation [23], DS was 2.99. In ESI-MS only peaks from completely alkylated cyclodextrins $1451.6[\mathrm{M}+\mathrm{Na}]^{+}, 737.4[\mathrm{M}+2 \mathrm{Na}]^{2+}$ for $\mathrm{Me}-\beta-$ $\mathrm{CD}$ and $1515.1[\mathrm{M}+\mathrm{Na}]^{+}, 769.1[\mathrm{M}+2 \mathrm{Na}]^{2+}$ for $\mathrm{Me}-d_{3}-\beta-\mathrm{CD}$, were detected.

\subsection{Partial hydrolysis}

Peralkylated- $\beta$-cyclodextrins were submitted to partial hydrolysis in a $1 \mathrm{~mL} \mathrm{~V}$-Vial. Ca. $2 \mathrm{mg}$ in $1 \mathrm{~mL}$ of $1 \mathrm{M}$ TFA was heated at $120{ }^{\circ} \mathrm{C}$ for $21(\mathrm{Me}-\beta-\mathrm{CD})$ and $15 \mathrm{~min}\left(\mathrm{Me}-d_{3}-\beta-\mathrm{CD}\right)$, respectively. After cooling to room temperature, aqueous acid was removed in a stream of nitrogen and finally co-distilled with toluene and the remainder evaporated to dryness. The obtained $O$-methylated maltooligosaccharides of DP 1-7 were dissolved in $1 \mathrm{~mL}$ of methanol. Composition with respect to DP was determined after reductive amination (2.4) by HPLCUV-MS (2.5) for each sample.

\subsection{Reductive amination}

The $O$-methylated maltooligosaccharides obtained by partial hydrolysis (2.2) were labeled [24] with $m$-aminobenzoic acid (mABA). To ca. $2 \mathrm{mg}$ of the substrate in $0.5 \mathrm{~mL} \mathrm{MeOH}$, $0.3 \mathrm{~mL} m \mathrm{ABA}$ in methanol (containing $2.6 \mathrm{mg} / 19 \mu \mathrm{mol} \mathrm{mABA}$ ) and $0.15 \mathrm{~mL}$ glacial acetic acid was added, and the mixture was heated to $40{ }^{\circ} \mathrm{C}$ for $30 \mathrm{~min}$. Subsequently, $50 \mu \mathrm{L}$ of

2-picoline borane in $\mathrm{MeOH}$ (containing $1.7 \mathrm{mg} ; \sim 19 \mu \mathrm{mol}$ ) were added, and the mixture was

heated to $40{ }^{\circ} \mathrm{C}$ for $45 \mathrm{~min}$. Subsequently the solvent was removed in a stream of nitrogen and the residue was dissolved in $1 \mathrm{~mL}$ of $\mathrm{MeOH}$. The completeness of labeling was determined by TLC and by ESI-IT-MS in positive ion mode for the presence of unlabeled sample. 


\subsection{HPLC-UV-MS}

LC-MS separations were carried out on an Agilent HPLC system consisting of a binary pump (1100 series) and a DAD (G1312A). A RP-C 18 -column (Phenomenex, Gemini, 250 x 4.60 $\mathrm{mm}, 5$ micron) was used. The mobile phase consisted of acetonitrile (ACN) and water with $1 \%$ added acetic acid [10]. Three different linear gradient systems were used starting with $\mathrm{H}_{2} \mathrm{O} / \mathrm{ACN} 90 / 10,80 / 20$ or $70 / 30(\mathrm{v} / \mathrm{v})$ at $t=0$ to $100 \% \mathrm{ACN}$ at $t=50 \mathrm{~min}$., respectively. If not otherwise stated, sample concentration was $0.2 \mathrm{mg} \mathrm{mL}^{-1}$ in $\mathrm{MeOH}$. Flow rate was set to $0.4 \mathrm{~mL} \mathrm{~min}{ }^{-1}$. UV absorption was recorded at $330 \mathrm{~nm}$ [10]. Mass spectra were obtained by coupling with ESI-IT-MS (2.7.1). Spectra were recorded in both, positive and negative ion mode. The parameters were the same as for syringe pump infusions except for the solvent (eluent of HPLC), nitrogen dry gas $\left(9 \mathrm{~L} \mathrm{~min}^{-1}, 365^{\circ} \mathrm{C}\right)$ and nebulizer gas (40 psi).

\subsection{Preparative HPLC}

Individual $O$-methylated maltooligosaccharide derivatives DP 1-7 were isolated from the partially hydrolyzed alkylated $\beta$-cyclodextrins by preparative HPLC (Knauer, well chrome) equipped with pump K-1001. The separation was carried out on a $\mathrm{RP}_{-} \mathrm{C}_{18}$-column (Phenomenex, Luna, 5 micron, 250x15.00 mm) at a flow rate of $5 \mathrm{~mL} \mathrm{~min}{ }^{-1}$. Mobile phase consisted of $\mathrm{H}_{2} \mathrm{O} / \mathrm{ACN}$, starting with $80 / 20(\mathrm{v} / \mathrm{v})$ at $t=0$, and reaching $100 \% \mathrm{ACN}$ at $t=50 \mathrm{~min}$. Sample concentration was $20 \mathrm{mg} \mathrm{mL}^{-1}$ and an injection volume of $200 \mu \mathrm{L}$ was used. As non-labeled compounds are not UV-active, the eluent from the column was collected every one minute in separate tubes. Fractions were monitored by TLC, and oligosaccharides of the same DP were combined, evaporated, and the residue dissolved in $\mathrm{MeOH}$. Results were further confirmed by syringe pump infusions of the unified fractions in the ESI-IT-MS instrument. In addition, the individual concentration of each DP in combined solutions was determined by HPLC-UV (2.5) after reductive amination (2.4) of an aliquot of the solution. 


\subsection{Mass spectrometry}

\subsubsection{ESI-IT-MS}

HCT Ultra ETDII (Bruker Daltonics, Bremen, Germany), equipped with an ion trap (IT), was employed. Syringe pump infusions were performed with analyte solution in methanol at a flow rate of $200 \mu \mathrm{L} \mathrm{h}^{-1}$. Nitrogen was used as dry gas $\left(4 \mathrm{~L} \mathrm{~min}^{-1}, 300{ }^{\circ} \mathrm{C}\right)$ and nebulizer gas (10 psi). Data was recorded in positive ion mode and the analytes were detected as sodium adducts. The following parameters were used: capillary $4.5 \mathrm{kV}$, end plate offset $500 \mathrm{~V}$, smart target 100,000 , trap drive level $100 \%$, compound stability $1000 \%$, capillary exit $280 \mathrm{~V}$ and skimmer $40 \mathrm{~V}$. If not otherwise stated, target mass was set to 1000 . These parameters are referred as 'defined (Standard?) conditions'.

\subsubsection{Nano-ESI-MS}

Thermo Scientific LTQ Orbitrap Velos Pro mass spectrometer equipped with both, orbitrap and linear ion trap was applied. Measurements were recorded in positive ion mode, both under orbitrap and ion trap conditions. Ion source parameters: capillary temperature, $300{ }^{\circ} \mathrm{C}$; spray voltage, $1 \mathrm{kV}$; spray current, $0.06 \mu \mathrm{A}$. Resolution was set to 60,000 with full scan screening, in the mass range $\mathrm{m} / \mathrm{z} 200-2000$, with a scan time of 10 microscans; source fragmentation 'on' and energy (V) was set to $100 \%$. Samples were dissolved in $\mathrm{MeOH}$ and each measurement was recorded for one minute.

\subsection{Mixtures of $O$-methyl maltooligosaccharides}

To study various parametrical influences on relative ion intensities (Rel. Int.) of $O$-deuteriomethylated $\left(\mathrm{Me}-d_{3}\right)$ and the corresponding $O$-methylated maltooligosaccharides (Me) in the range of DP 2-6, two types of standard mixtures of varying molar composition were prepared, binary mixtures (BM) and complex mixtures (CM). 
$O$-Methylated maltooligosaccharides (Me and Me- $d_{3}$ ) of individual DP obtained according to 2.2, 2.3 and 2.6 were dissolved in $\mathrm{MeOH}$ at a concentration of about $2 \mathrm{mg} \mathrm{mL}^{-1}$. A portion of each DP solution was reductively aminated (2.4), and the exact concentration of individual DP was determined by HPLC-UV (2.5). Unlabeled Me and Me- $d_{3}$ oligomers of same DP were mixed in certain proportion to prepare binary standard mixtures with defined molar ratio (MR) of the two components. Later, a portion of these standard binary mixtures was reductively aminated (2.4) in triplicates to determine the exact molar ratio by HPLC-UV (2.5).

\subsubsection{Complex Mixtures (CM)}

Certain volumes, calculated on the basis of their composition (2.5) of the solutions of partially hydrolyzed Me- $\beta-\mathrm{CD}$ and Me- $d_{3}-\beta-\mathrm{CD}$ (2.3), were combined to obtain complex mixtures with defined MR of each DP of the two types of $O$-methylated oligosaccharides (Me and Me- $d_{3}$ ), respectively. Similar to BM, exact MRs of the constituents of each CM were determined in triplicates after reductive amination.

\subsection{Data processing}

All mass spectral data were analyzed by Bruker Daltonics Data Analysis software. Peak intensities in mass spectra were analyzed by adding the relative intensity of the $1^{\text {st }}$ to $5^{\text {th }}$ calculated isotopic signal to the main peak. 


\section{RESULTS AND DISCUSSION}

\subsection{Molar ratio of standard mixtures}

To study the influence of chemistry and size of analog oligosaccharide derivatives on their ion yield in ESI-IT-MS, binary mixtures $(\mathrm{BM})$ of known concentration and molar ratio were prepared from $O$-methylated and $O$-deuteromethylated maltooligosaccharides. In addition, complex mixtures $(\mathrm{CM})$ containing the entire range of oligomers obtained from $\beta-\mathrm{CD}$ by partial hydrolysis were employed. These mixtures resemble the complexity of samples measured in the 'oligomer analysis' of polysaccharide ethers, to gain insight in the substituent distribution on the polymer level $[4,10]$. All mixtures prepared from defined stock solutions were finally analyzed with respect to their real molar ratios of the corresponding oligomers $\left(\left[\mathrm{Me}-d_{3}\right] /[\mathrm{Me}]\right)$ by HPLC/UV after reductive amination (2.5). Data are given in Table 1 and Table 2 for BM and various CM, respectively.

\section{Table 1}

Molar ratio of oligomers $\left(\left[\mathrm{Me}-d_{3}\right] /[\mathrm{Me}]\right)$ in individual BM, determined by HPLC-UV ( $\mathrm{n}=3$ ), given with uncertainty

\begin{tabular}{cccccc}
\hline BM & DP 2 & DP 3 & DP 4 & DP 5 & DP 6 \\
\hline 1 & $0.77 \pm 0.002$ & $0.84 \pm 0.001$ & $1.05 \pm 0.003$ & $1.28 \pm 0.002$ & $1.06 \pm 0.001$ \\
2 & $1.01 \pm 0.007$ & $0.80 \pm 0.001$ & $2.44 \pm 0.001$ & $1.21 \pm 0.001$ & $1.05 \pm 0.003$ \\
\hline
\end{tabular}

*DP: Degree of polymerization

\section{Table 2}

Molar ratio of oligomers $\left(\left[\mathrm{Me}-d_{3}\right] /[\mathrm{Me}]\right)$ in individual $\mathrm{CM}$ of $O$-methylated maltooligosaccharides, determined by HPLC-UV after reductive amination $(n=3)$, given with uncertainty

\begin{tabular}{cccccc}
\hline $\mathbf{C}$ & DP2 & DP3 & DP4 & DP5 & DP6 \\
$\mathbf{M}$ & & & & & \\
\hline 1 & $0.94 \pm 0.004$ & $0.72 \pm 0.003$ & $0.67 \pm 0.002$ & $0.53 \pm 0.002$ & $0.38 \pm 0.001$ \\
2 & $1.09 \pm 0.015$ & $1.22 \pm 0.027$ & $1.30 \pm 0.028$ & $1.48 \pm 0.020$ & $1.74 \pm 0.024$ \\
3 & $1.21 \pm 0.001$ & $1.36 \pm 0.001$ & $1.44 \pm 0.001$ & $1.65 \pm 0.001$ & $1.93 \pm 0.001$ \\
4 & $1.22 \pm 0.014$ & $1.37 \pm 0.026$ & $1.46 \pm 0.027$ & $1.66 \pm 0.020$ & $1.95 \pm 0.023$ \\
5 & $1.37 \pm 0.029$ & $1.06 \pm 0.015$ & $0.98 \pm 0.014$ & $0.77 \pm 0.011$ & $0.55 \pm 0.018$ \\
6 & $1.37 \pm 0.019$ & $1.54 \pm 0.010$ & $1.63 \pm 0.011$ & $1.87 \pm 0.012$ & $2.19 \pm 0.014$ \\
7 & $1.54 \pm 0.017$ & $1.19 \pm 0.015$ & $1.10 \pm 0.005$ & $0.87 \pm 0.004$ & $0.63 \pm 0.003$ \\
8 & $1.57 \pm 0.014$ & $1.77 \pm 0.026$ & $1.87 \pm 0.027$ & $2.14 \pm 0.029$ & $2.51 \pm 0.022$ \\
9 & $1.78 \pm 0.032$ & $2.00 \pm 0.046$ & $2.12 \pm 0.048$ & $2.43 \pm 0.044$ & $2.85 \pm 0.051$ \\
\hline
\end{tabular}

${ }^{*} \mathrm{CM} 2,3,4,6,8$ and 9 and $\mathrm{CM} 1,5$ and 7 are prepared from two different sets of stock solutions of individual oligomer solution of Me and Me- $d_{3}$. 


\subsection{Normalization of data}

As shown in Tables 1 and 2, the molar ratio MR with respect to each DP is known for all mixtures used in this study. Rel. Int. ( $\left(\mathrm{I}_{\mathrm{Me}-d 3} / \mathrm{I}_{\mathrm{Me}}\right)$ obtained from ESI-IT-MS measurements of mixtures with different $M R$ are normalized to equimolar concentrations $(M R=1)$ for each individual DP. Normalized Rel. Int., which represents the relative sensitivities of $\mathrm{Me}-d_{3} / \mathrm{Me}$ will be denoted as $\left(\mathrm{I}_{\mathrm{Me}-d /} / \mathrm{I}_{\mathrm{Me}}\right) / \mathrm{MR}$. For a robust quantitative ESI-IT-MS method, relative sensitivities $\left[\left(\mathrm{I}_{\mathrm{Me}-d 3} / \mathrm{I}_{\mathrm{Me}}\right) / \mathrm{MR}\right]$ should be constant under the instrumental conditions and within the range of sample concentrations applied.

\subsection{Dependence of relative sensitivities on molar ratio of analytes}

A prerequisite for quantification of MR of two analytes is a linear relationship between MR $\left(\left[\mathrm{Me}-d_{3}\right] /[\mathrm{Me}]\right)$ of the sample and the signal $\left(\mathrm{I}_{\mathrm{Me}-\mathrm{d} 3} / \mathrm{I}_{\mathrm{Me}}\right)$. In 'oligomer analysis' of polysaccharide derivatives, MR of analytes of a certain DP with various substituent patterns present in different proportions must be determined [10]. Therefore, we proved that relative sensitivity increased linearly with the increase of the MR $\left(\left[\mathrm{Me}-d_{3}\right] /[\mathrm{Me}]\right)$ of the analytes in the sample solution. According to the literature [27], the surface of the droplet formed in the electrospray process is usually saturated at common sample concentrations. Consequently, there is a competition between various analyte molecules for places on this surface from where they can be evaporated to form gas phase ions after desolvation (according to the ion evaporation model). This competition between analyte molecules could affect the ionizing efficiency of different analyte molecules which will in turn influence the Rel. Int. in ESI-MS. Therefore, we performed a study with increasing MR of the samples, at a total concentration range of $10^{-6}$ to $10^{-5} \mathrm{M}$, the critical range for surface saturation [27].

We started our study with binary mixtures (BM) containing only one DP of each type, and later extended the study to complex mixtures containing all DPs of interest (CM). Initially BM of DP 2 and DP 5 were prepared with increasing MR, to check linearity and to investigate 
whether the relative sensitivity varies with DP. Fig. 1a shows that both oligomers display linearity with increase of MR $\left(\left[\mathrm{Me}-d_{3}\right] /[\mathrm{Me}]\right)$ in this range of concentration. Results for all the BM in Table 1 are shown in Fig. 1b. A decrease of the relative sensitivity with increasing DP was observed. With 1.01 for DP2, $\left(\mathrm{I}_{\mathrm{Me}-d 3} / \mathrm{I}_{\mathrm{Me}}\right) / \mathrm{MR}$ was equal as expected for these chemically very similar compounds, but was found to be only 0.78 for DP 6 . To extend these studies to more complex mixtures, all CMs shown in Table 2 were analyzed at a total concentration of $10^{-6} \mathrm{M}$. to study the effect of saturation due to competition from other DPs. It is observed from the results (Fig. 2) that the relative sensitivity of the DP of interest is not influenced by the presence of other DPs and is linearly proportional to increasing MR ([Me- $\left.\left.d_{3}\right] /[\mathrm{Me}]\right)$. From the various slopes of the graphs in Fig. 2 dependence of relative sensitivities in CM on DP is visible. With 1.09 for DP 2 it is slightly higher than the expected 1.0 observed in BM. As for the $\mathrm{BM}$, the relative sensitivity decreased with DP over 0.98 for DP 3, 0.96 for DP4, and 0.87 for DP 5 to only 0.71 for DP 6 (see 3.5).

Tang and Kebarle [28] used the relationship (equation 1) between relative ion intensities $\left(I_{\mathrm{A}} / I_{\mathrm{B}}\right)$, relative sensitivity coefficients $\left(k_{\mathrm{A}} / k_{\mathrm{B}}\right)$, and molar ratio of components ([A]/ [B]) for a two-component system with the presence of electrolytes.

$$
\frac{I_{A}}{I_{B}}=\frac{k_{A}[A]}{k_{B}[B]}
$$

In Fig. 1 and Fig. 2 it can be observed that relative ion intensities increase linearly with molar ratio for all DPs in BM and CM, while the relative sensitivity is decreasing with DP (investigated in later sections). These results imply that molar ratio can be quantified from relative ion intensities as long as the relative sensitivity coefficient of the components is known. Robustness of relative sensitivities $\left(k_{\mathrm{A}} / k_{\mathrm{B}}\right)$ were further studied with regard to the influence of total concentration of the sample, while keeping the MR constant (3.5). 
a)

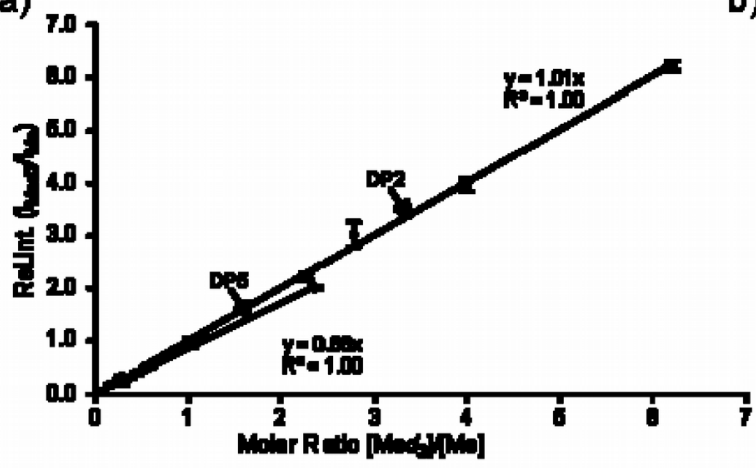

b)

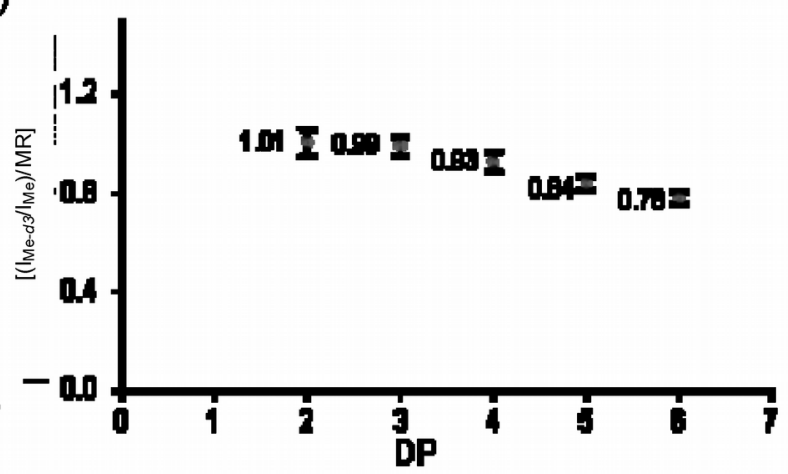

Fig. 1. a) Measured relative ion intensities with increasing MR ([Med $\left.\left.d_{3}\right] /[\mathrm{Me}]\right)$ in BM of DP 2 and DP 5 ; each data point is an average of three measurements. The slope gives the relative sensitivity. b) Average of all measurements of relative sensitivities $\left[\left(\mathrm{I}_{\mathrm{Me}-\mathrm{d} / 3} / \mathrm{I}_{\mathrm{Me}}\right) / \mathrm{MR}\right]$ of BM 1 and BM 2; each DP in BM 1 and BM 2 is measured 10 times, error bars show the uncertainty limits with 95\% confidence (3.4, Table 3). Samples were measured in positive ion mode under conditions defined in 2.7.1, at a total concentration range of the sample from $10^{-6}$ to $10^{-5} \mathrm{M}$ in methanol. 


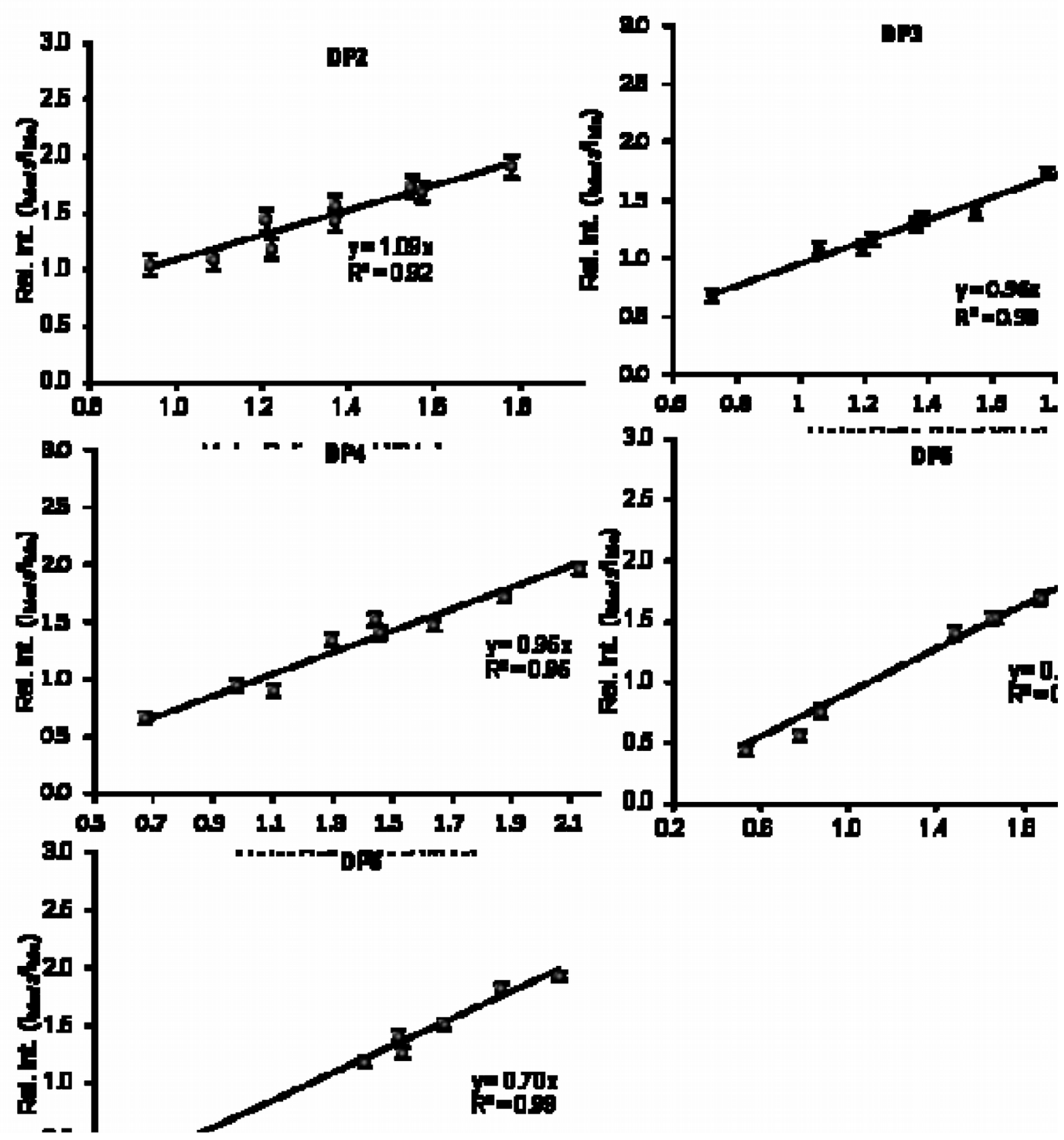

Fig. 2. Relative ion intensities with increase in MR ([Me- $\left.\left.d_{3}\right] /[\mathrm{Me}]\right)$ in CM (Table 2) for DP 2-6. Samples were measured in positive ion mode under conditions defined in 2.7.1; each data point is an average of three syringe pump infusion measurements at a total concentration of $10^{-6} \mathrm{M}$ in methanol. The slopes represent the relative sensitivities.

\subsection{Measurement uncertainty}

All measurements described above were performed in our laboratory with a Bruker HCT ultra ESI-IT-MS instrument, which is intensively used by several research groups for different applications. Since the condition of the instrument is critical with respect to reproducibility of absolute ion intensities, measurement uncertainty was determined for all $\mathrm{BM}$ and for $\mathrm{CM}$ (with $\mathrm{CM} 4$ ) to differentiate the parametrical influence on Rel. Int. from that caused by the 
instrument. Hence, a study was performed by syringe pump infusion, five consecutive times each day and on five different days under defined conditions (2.7.1). Uncertainty $\left(\mathrm{u}_{1}\right)$ was calculated for each DP both in BM and CM. This uncertainty $\left(\mathrm{u}_{1}\right)$ was combined with uncertainty $\left(\mathrm{u}_{2}\right)$ from the determination of reference data by HPLC-UV (3.1), and a combined uncertainty (U) with $95 \%$ confidence was calculated by following 'Type A evaluation' $[25,26]$. $\mathrm{U}$ values for all the DPs in BM and CM are given in Table 3 (3.5).

\subsection{Effect of total concentration on relative sensitivities}

Tang and Kebarle (1993) [28] and Kebarle and Verkerk (2009) [29] reported on relative sensitivity coefficients (as given in equation 1) of two components with similar, slightly different, and very different relative sensitivities in ESI-MS over a wide range of total concentration $\left(10^{-8}\right.$ to $\left.10^{-2} \mathrm{M}\right)$, while keeping the molar ratio of the components equal $([\mathrm{A}]=[\mathrm{B}])$. Up to a total concentration of about $10^{-6} \mathrm{M}$, they observed a linear increase of the ion intensities. At higher total concentration, absolute intensities did not increase further, but the ratio of sensitivity coefficients changed and remained constant above $10^{-5} \mathrm{M}$. This behavior was explained by saturation of the droplet surface and a continuous re-supply from the bulk of the droplet after the depletion of ions at the surface. Analytes with lower ionizing efficiency have a lower chance to reach the surface and to survive the subsequent fission. In contrast, at lower concentration all analyte molecules have a chance to escape from droplet and form ions, because the surface is not yet saturated. This phenomenon leads to a change of observed relative sensitivity coefficients. Tang and Kebarle [28] reported that Rel. Int. of two analytes with different sensitivities in ESI represent their sensitivity coefficients when competing for the droplet surface at higher concentration; at lower concentrations these may approach 1. Earlier studies have been performed with simple alkali ions, quaternary ammonium ions and alkaloids. In our typical application, the analysis of oligosaccharide derivatives, obtained from corresponding polysaccharides, we are confronted with a complex mixture with varying MRs with respect to DP and to chemistry within an individual DP. 
Hence, the dependence of Rel. Int. on total concentration and MRs of components has to be studied in detail.

Initially, DP2 and DP5 of BM 1 (Table 1) were gradually diluted from a concentration of $10^{-5}$ to $10^{-9} \mathrm{M}$, in addition, CM 9 (Table 2) was gradually diluted from $10^{-5}$ to $10^{-8} \mathrm{M}$. Results of ESI-MS of BM and CM are shown in Fig. 3 and Fig. 4, respectively. From these Figures, it can be observed that the absolute ion intensities of Me and Me- $d_{3}$ oligomers in BM and $\mathrm{CM}$ exhibit a flattening effect after $10^{-6} \mathrm{M}$. This effect is more pronounced in $\mathrm{CM}$ compared to BM, which is expected due to competition from other DPs in CM. Nevertheless, the decreasing trend of relative sensitivities with an increase in DP does not seem to improve irrespective of total concentration in both BM and CM.
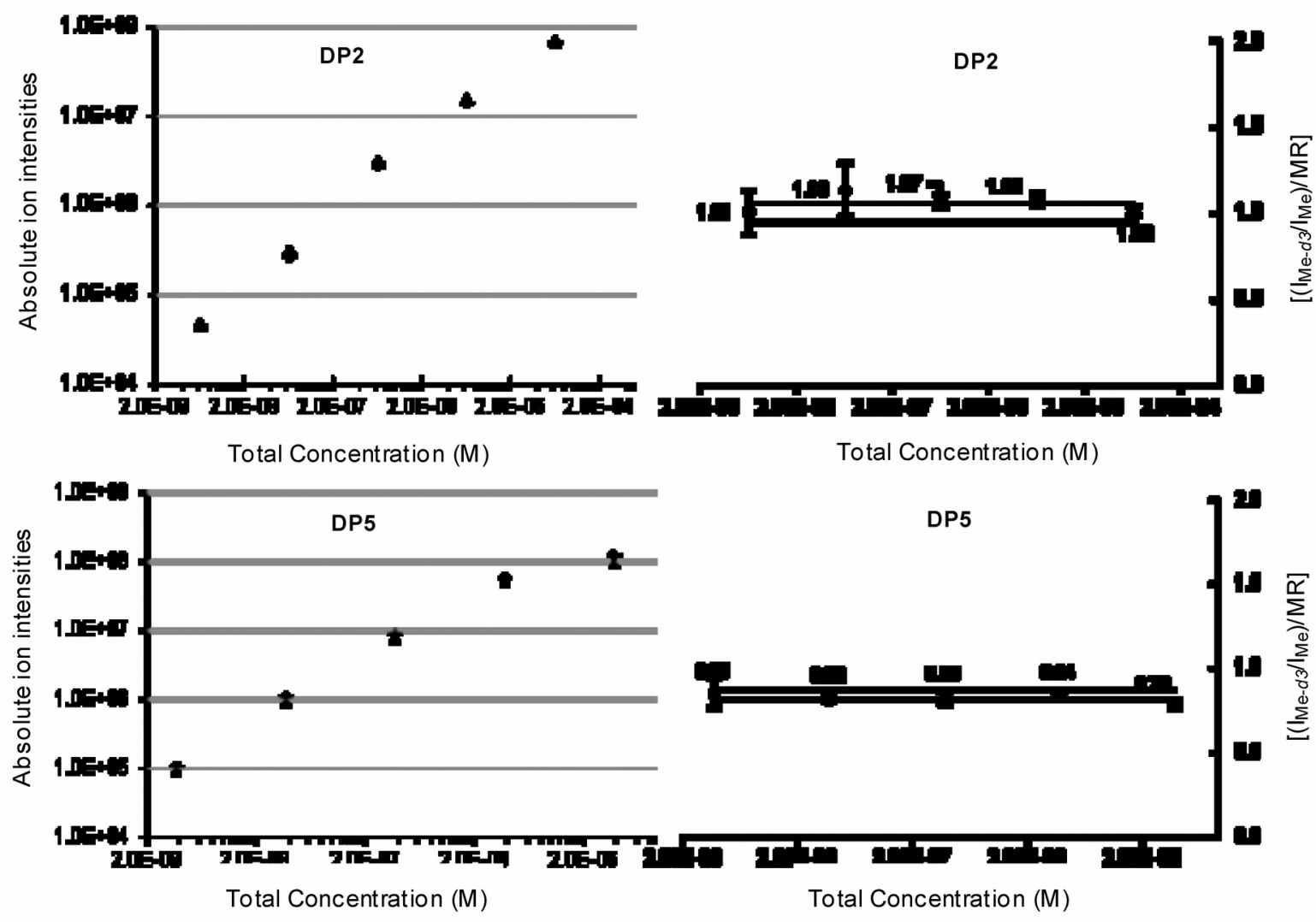

Fig. 3. BM 1 (Table 1) of DP2 and DP5; measured at varying total concentration of BM while keeping the MR constant; conditions see 2.7.1. Right-absolute ion intensities (normalized to an equimolar ratio), left-relative sensitivities, at different total concentrations; given with the average values shown in fig. $1 \mathrm{~b}$ and uncertainty limits $(3.4$, Table 3$)$, each data point shows the standard deviation of $n=3$. 


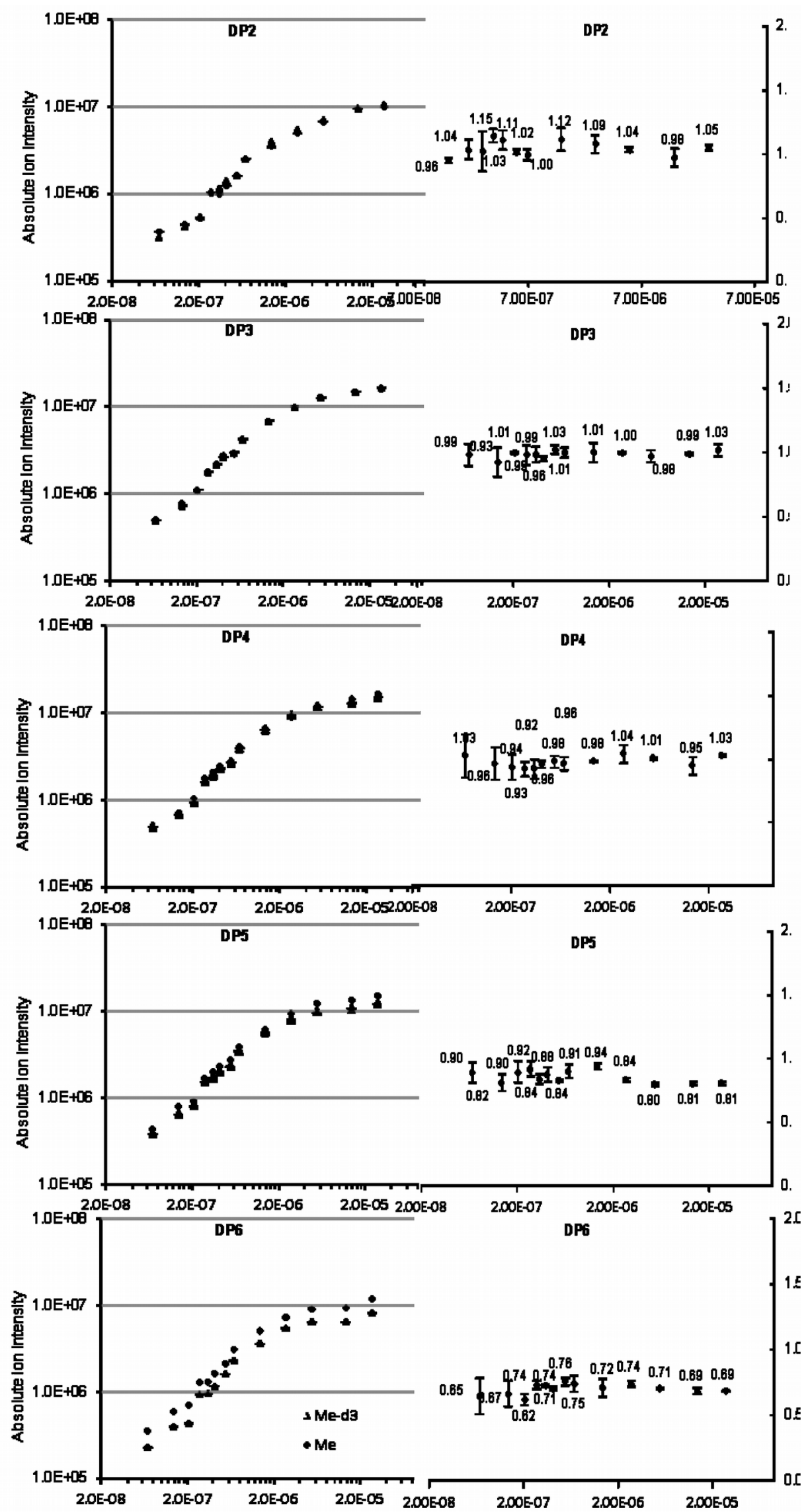

Fig.4. CM 9 (for composition see Table 2); Left: Absolute ion intensities (normalized to an equimolar ratio), of oligomers of different DP measured at varying total concentration in methanol, while the MR of the components is kept constant; Right: relative sensitivity of each DP; conditions see 2.7.1; given with the average relative sensitivities and U limits $95 \%$ confidence (3.4, Table 3), each data point shows the standard deviation of $\mathrm{n}=3$. 
At lower concentration and increasing the $\mathrm{S} / \mathrm{N}$ ratio, the standard deviation of the measurement increases as expected, but the relative sensitivities of these very similar analytes are not concentration dependent in this concentration range, but are constant. This confirms that the ESI-IT-MS method can be applied for the quantification of MR in complex mixtures, typical for the analysis of substituent patterns in polysaccharide derivatives. The deviation from the expected equal ion yield and the drop of relative sensitivity coefficients $\left(\mathrm{k}_{\mathrm{Me}-\mathrm{d} 3} / \mathrm{k}_{\mathrm{Me}}\right)$ with increasing DP was, however, unexpected. No such discrimination effect is observed for the randomly substituted $O$-Me/O-Me- $d_{3}$-oligosaccharides obtained from partial hydrolysis of methyl cellulose. In contrast to our model samples with the extreme cases of fully $O$-methylated and fully $O$-deuteromethylated oligosaccharides, oligosaccharides obtained from deuteromethylated methyl cellulose contain all possible patterns, e.g. in case of DP 3 from $\mathrm{Me}_{9} /\left(\mathrm{Me}-d_{3}\right)_{0}$ over $\mathrm{Me}_{8} /\left(\mathrm{Me}-d_{3}\right)_{1}, \mathrm{Me}_{7} /\left(\mathrm{Me}-d_{3}\right)_{2}$ etc. up to $\mathrm{Me}_{0} /\left(\mathrm{Me}-d_{3}\right)_{9}$. With increasing DP these profiles become narrower, and thus the analytes being compared become more similar in mass and polarity. Thus, the drop of relative sensitivity coefficients $\left(\mathrm{k}_{\mathrm{Me}-\mathrm{d} 3} / \mathrm{k}_{\mathrm{Me}}\right)$ with increasing DP is probably of low relevance for randomly substituted polymer derivatives [10], but have to be considered in case of block like structures [30]. Moreover, it is of interest to understand the reason behind this behavior. Average values for all DPs of BM ( $n=20$ for each DP) and $\mathrm{CM}(\mathrm{n}=108)$ mixtures (Table 2) which were measured at three different total concentrations of the sample in the range $10^{-6}$ to $10^{-5} \mathrm{M}$ with $\mathrm{U}$ limits (see 3.4) are given in Table 3.

\section{Table 3}

Relative sensitivities $\left[\left(\mathrm{I}_{\mathrm{Me}-d 3} / \mathrm{I}_{\mathrm{Me}}\right) / \mathrm{MR}\right]$ of $\mathrm{BM}$ and $\mathrm{CM}$ given with $\mathrm{U}$ limits $(95 \%$ confidence)

\begin{tabular}{cccccc}
\hline $\begin{array}{c}\text { Mol. Rel. } \\
\text { Int. }\end{array}$ & DP 2 & DP 3 & DP 4 & DP 5 & DP 6 \\
\hline BM & $1.01 \pm 0.04$ & $0.99 \pm 0.03$ & $0.93 \pm 0.03$ & $0.84 \pm 0.02$ & $0.78 \pm 0.02$ \\
CM & $1.06 \pm 0.09$ & $0.98 \pm 0.06$ & $0.96 \pm 0.06$ & $0.87 \pm 0.05$ & $0.71 \pm 0.05$ \\
\hline
\end{tabular}

The data shows that there is no significant difference in the relative sensitivities of deuteromethylated and methylated maltooligosaccharides measured in binary and complex 
mixtures. Standard deviation is lower for binary mixtures (2-4\%) compared to the complex mixtures $(6-9 \%)$.

\subsection{Effect of DP on molar relative intensities of Me and Me- $d_{3}$ oligosaccharides}

It can be derived from Figs. 1-4 that relative sensitivities $\left[\left(\mathrm{I}_{\mathrm{Me}-\mathrm{d} 3} / \mathrm{I}_{\mathrm{Me}}\right) / \mathrm{MR}\right]$ are decreasing with DP. As DP increases, the chemical character of the homolog oligosaccharide ethers in principle remains the same, but the absolute size and mass difference between two corresponding oligomers of Me- and Me- $d_{3}$-series increases. The chance of an analyte to be located on the droplet surface, is assumed to depend on its surface activity and electrophoretic mobility. Compared to $\mathrm{CH}_{3}$, the $\mathrm{CD}_{3}$ group is more hydrophobic, more volatile and probably more easily desolvated in $\mathrm{MeOH}$. These properties favor a higher ionization rate and thus a relative sensitivity $\left[\left(\mathrm{I}_{\mathrm{Me}-\mathrm{d} 3} / \mathrm{I}_{\mathrm{Me}}\right) / \mathrm{MR}\right]>1$. This is indeed observed in CM for DP 2, where the value is slightly above an equal intensity. Already at DP 3 , however, $\left(\mathrm{I}_{\mathrm{Me}-d 3} / \mathrm{I}_{\mathrm{Me}}\right) / \mathrm{MR}$ drops below 1.00 and further decreases to about 0.87 for DP 5 and even 0.71 for DP 6 (see Table 3). Obviously, another effect begins to dominate. The deuteromethyl group is bulkier and higher in mass than methyl and this mass difference increases by $9 \mathrm{Da} /$ glucosyl unit. Consequently, the differences in electrophoretic mobility - at same charge - will increase with DP in favor of the smaller Me oligomers. Literature for this phenomenon in ESI is rare. A Monte Carlo simulation study has been performed which predicts that 'analytes with higher molecular weight and larger size have lower ionizing efficiency than smaller molecules' [32]. Therefore, the initial droplet size generated in the electrospray ionization process should also influence the relative ion intensities. To investigate this effect, we applied nano-ESI-MS for comparison (see below, 3.7).

\subsection{Nano ESI-MS}

Nano-ESI-MS is known to reduce discrimination in the ionization process. Due to the small diameter of the spraying capillary, initial droplet size is reduced, which means more surface and less bulk, fewer generations of Coulomb explosions and thus less discrimination of the 
less surface active and slower migrating compounds. This phenomenon was impressively demonstrated for a mixture of maltopentaose and the protein insulin by Bahr \& Karas [18]. At a very low diameter of the spray capillary and thus droplet size less than $200 \mathrm{~nm}$, i.e., 100 1000 times smaller than normal ESI, discrimination due to different chemical behavior and size can be suppressed [33]. To establish whether this effect can be observed for $\mathrm{Me} / \mathrm{Me}-d_{3}$ mixtures, binary mixtures (BM 2, Table 1) were measured in nano-ESI-IT-MS and nano-ESIOrbitrap-MS under the conditions reported in 2.7.2. Fig. 5 depicts the results obtained from nano-ESI measurements of both, ion trap and orbitrap-MS. Indeed, no decrease of relative sensitivities with an increase in DP is observed, indicating that the differences in surface activity and electrophoretic mobility no longer play any role. Rel. Int. for $O$-Me- $d_{3} / O$-Me-maltooligomers are scattered around 1.0 without any trend with an average value of 1.01 for all DPs; with normal ESI-IT-MS they decrease from 1.01 at DP2 to 0.78 at DP6 for BM as shown above. There is no systematic difference between the results obtained by nano-ESI-IT-MS and nano-ESI-Orbitrap-MS.
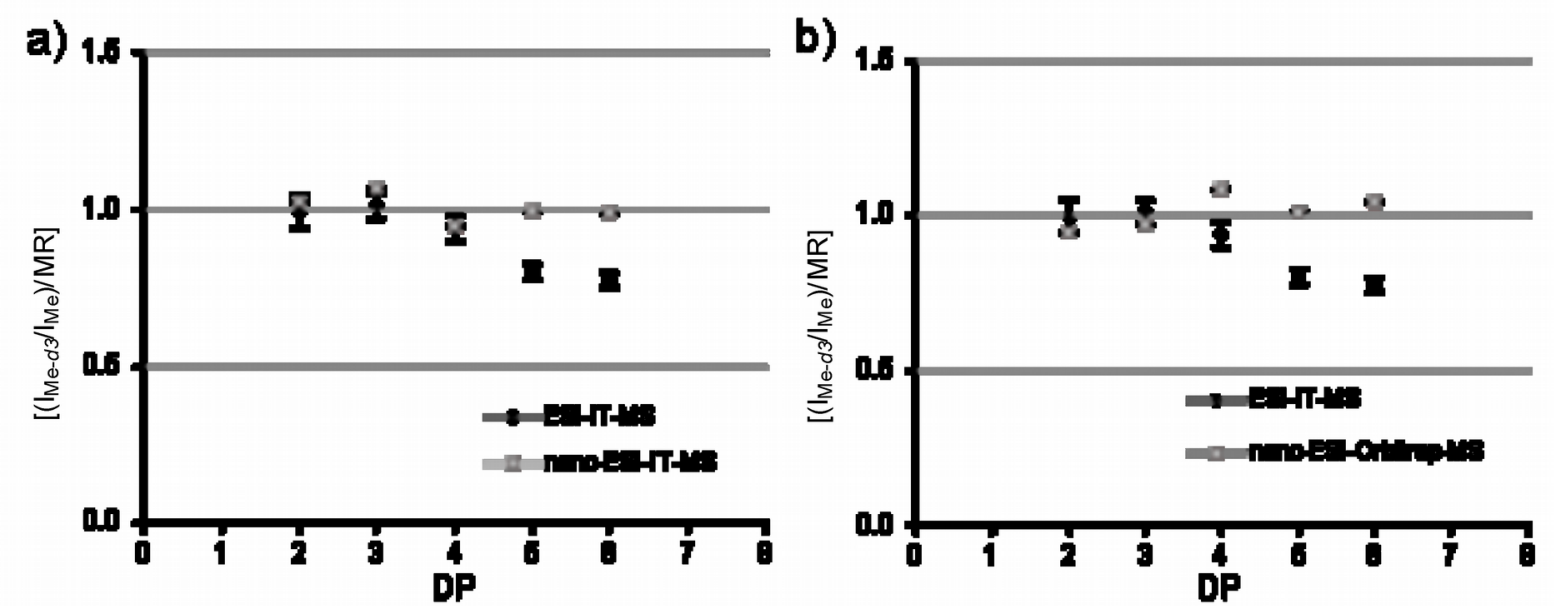

Fig. 5. a) Comparison of relative sensitivities of BM 2 from Table 1 measured in ESI-IT-MS and nano-ESI-IT-MS. b) Comparison of relative sensitivities of BM 2 from Table 1 measured in ESI-IT-MS and nano-ESI-Orbitrap-MS. Samples were dissolved in methanol at a total concentration of ca. $10^{-6} \mathrm{M}$, measured under the conditions given in 2.7.1 and 2.7.2 for ESI-MS and nano-ESI-MS, respectively. 


\subsection{ESI-IT-MS of labeled oligosaccharides}

Differences in ionization efficiency of analytes of different chemistry and size can also be overcome by labeling [34]. The introduction of a charged tag, amino benzoic acid for negative ion mode, and Girard's T for positive ion mode [31], can level off differences in sodium complexation ability and at the same time influence surface activity. oABA-labeled $O$-Me/O-Me- $d_{3}$ cellooligosaccharides have been analyzed by LC-ESI-IT-MS up to DP 10 by Cuers et al. [10]. In the analysis of such real samples, analytes can cover the whole range of partially $O-\mathrm{Me} / O-\mathrm{Me}-d_{3}$ carbohydrates. In more or less randomly substituted polysaccharides small or - with increasing DP - no amounts of the extreme cases of fully methylated and fully deuteromethylated oligosaccharides are present, but should also be quantifiable in case of unusual, e.g. bimodal or blocky patterns [30]. Therefore, we tested whether labeling can level off the decrease in relative sensitivity observed with the normal ESI-MS for oligomers of higher DP. Reductively aminated BM ( $m \mathrm{ABA}, 2.4)$ were measured in negative ion mode under the conditions mentioned in 2.5. Quantitative evaluation (Fig. 6) shows that the differences observed for corresponding higher Me and Me- $d_{3}$ oligomers can also be leveled off by this tag, and thus the range for quantitative evaluation in the analysis of the substitution patterns of polysaccharide derivatives is extended [10].

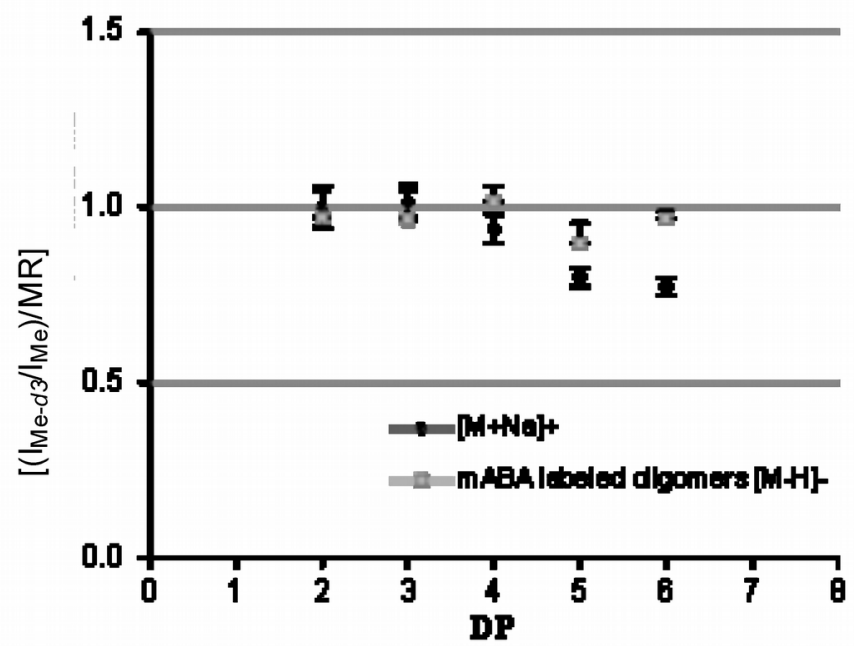

Fig. 6. Comparison of relative sensitivities of $\mathrm{BM} 2$ from Table 1 ; $[\mathrm{M}+\mathrm{Na}]^{+}$measured in positive ion mode and $[\mathrm{M}-\mathrm{H}]^{-}$of labeled oligosaccharides (reductively aminated with $m \mathrm{ABA}$ ) measured in negative ion mode. Samples were dissolved in methanol at a total 
concentration of approximately $10^{-5} \mathrm{M}$, measured under the conditions reported under 2.7 .1 and 2.5 respectively; error bars show the standard deviation, $\mathrm{n}=3$; target mass was 1000 in both cases.

\section{Conclusion}

The influence of various parameters on ion intensities of $O$-methylated maltooligosaccharides and their $O$-deuteromethylated isotopomers in ESI-MS has been investigated for binary and complex mixtures of DP 2 to 6 . Absolute ion intensities linearly increased with concentration until flattening due to saturation set in, while relative sensitivities remained constant. The relative sensitivity coefficients of Me- $d_{3} /$ Me derivatives, however, decreased with DP from values close to 1.0 for DP 2 to $0.7-0.8$ for DP 6 , i.e. with increasing mass and mass difference and without pronounced differences for binary and complex mixtures. The competition of analytes did not play an important role on relative sensitivity coefficients. Measurement of uncertainty including uncertainty of reference data of sample composition and of the instrument for repeated measurements were in the range of 3 to $9 \%$. Bias observed for the larger oligomers could be leveled by labeling with $m \mathrm{ABA}$, indicating that not the mass analysis by ion trap is responsible for discrimination but the ionization process, if parameters, especially target mass are properly adjusted. Differences in relative sensitivities could also be overcome by using nano-ESI-MS, both with ion trap and orbitrap. This confirms that smaller droplet size and fewer generations of Coulomb fissions reduce or eliminate sources of discrimination related to surface activity and electrophoretic mobility of the analytes. With respect to the application of ESI-MS analysis of oligosaccharide ethers in the field of polysaccharides derivatives, we proved its reliability under appropriate conditions. The quantification of the extreme cases, the fully methylated and fully deuteromethylated oligomers relevant in bimodal and block like substituted derivatives becomes critical at DP 4 if normal ESI-MS without labeling is applied. To gain more insight into the factors influencing relative ion intensities in ESI extended studies with other, more different types of oligosaccharide derivatives are in progress. 


\section{ACKNOWLEDGEMENT}

We gratefully acknowledge support by the Braunschweig International Graduate School of Metrology (B-IGSM) and the DFG Research Training Group GrK1952/1 "Metrology for Complex Nanosystems". We are thankful to Mrs. Andrea Abrahamik for technical assistance with nano-ESI measurements at Helmholtz-Centre for Infection Research, Braunschweig.

\section{REFERENCES}

D. Klemm, B. Heublein, H. P. Fink, A. Bohn, Cellulose: fascinating biopolymer and sustainable raw material, Angew. Chem. Int. Ed. 44 (2005) 3358-3393.

[1] K. J. Edgar, Polysaccharide chemistry: frontiers and challenges. In: polysaccharide materials: performance by design. ACS symp ser 1017 (2010): pp. 1-12.

[2] P. Mischnick, C. Hennig, A new model for the substitution patterns in the polymer chain of polysaccharide derivatives, Biomacromolecules 2 (2001) 180-184.

[3] P. Mischnick, D. Momcilovic, Chemical structure analysis of starch and cellulose derivatives, Adv. Carbohydr. Chem. Biochem. 64 (2010) 117-210.

[4] P. Mischnick, Structural analysis of polysaccharides and polysaccharide derivatives, Macromol. Symp. 99 (1995) 3-13.

[5] R. Adden, C. Melander, G. Brinkmalm, L. Gorton, P. Mischnick, New approaches to the analysis of enzymatically hydrolyzed methyl cellulose. Part 1. Investigation of the influence of structural parameters on the extent of degradation, Biomacromolecules 7 (2006) 1399-1409.

[6] R. Adden, W. Niedner, R. Müller, P. Mischnick, Comprehensive analysis of the substituent distribution in the glucosyl units and along the polymer chain of hydroxyethylmethyl celluloses and statistical evaluation, Anal. Chem. 78 (2006) 1146-1157.

[7] P. Mischnick, G. Kühn, Model studies on methyl amyloses: correlation between reaction conditions and primary structure, Carbohydr. Res. 290 (1996) 199-207.

[8] R. Adden, R. Müller, P. Mischnick, Fractionation of methyl cellulose according to Polarity - a tool to differentiate first and second order heterogeneity of the substituent distribution, Macromol. Chem. Phys. 207 (2006) 954-965.

[9] J. Cuers, I. Unterieser, W. Burchard, R. Adden, M. Rinken, P. Mischnick, Simultaneous determination of substituent patterns in partially acid hydrolyzed $O-\mathrm{Me} / O-\mathrm{Me}-d_{3}$-cellulose and quantification of the obtained oligomers by HPLC-ESI-MS, Carbohydr. Res. 348 (2012) 55-63.

[10] P. Kebarle, M. Peschke, On the mechanisms by which the charged droplets produced by electrospray lead to gas phase ions, Anal. Chim. Acta. 406 (2000) 11-35.

[11] G. Taylor, Disintegration of Water Drops in an Electric Field, Math. Phys. Sci. 280 (1964) 383-397.

[12] M. S. Wilm, M. Mann, Electrospray and taylor-cone theory, Dole's beam of macromolecules at last?, Int. J. Mass Spectrom. 136 (1994) 167-180.

[13] J. B. Fenn, Ion formation from charged droplets: Roles of geometry, energy, and time, J. Am. Soc. Mass Spectrom. 4 (1993) 524-535.

[14] J. V. Iribarne, B. A. Thomson, On the evaporation of small ions from charged droplets, J. Chem. Phys. 64 (1976) 2287-2294.

[15] M. Dole, A. Rheude, L. L. Mack, Molecular beams of macroions. II, J. Chem. Phys. 52 (1970) 4977-4986.

[16] S. Nguyen, J. B. Fenn, Gas-phase ions of solute species from charged droplets of solutions, Proc. Natl. Acad. Sci. U. S. A. 104 (2007) 1111-1117.

[17] A. Schmidt, M. Karas, Effect of different solution flow rates on analyte ion signals in nano-ESI MS, or: When does ESI turn into nano-ESI?, J. Am. Soc. Mass Spectrom. 14 (2003) 492-500.

[18] U. Bahr, A. Pfenninger, M. Karas, High-sensitivity analysis of neutral underivatized oligosaccharides by nanoelectrospray mass spectrometry, Anal. Chem. 69 (1997) 4530-4535.

[19] B. N. Pramanik, A. K. Ganguly, M. L. Gross, Applied electrospray mass spectrometry, 2002.

[20] I. Ciucanu, F. Kerek, A simple and rapid method for the permethylation of carbohydrates, Carbohydr. Res. 131 (1984) 209-217. 
[21] J. R. Johnson, N. Shankland, I.H. Sadler, Full assignment of the proton and carbon-13 NMR spectra of 2,3,6-tri- $O$-methyl- $\beta$-cyclodextrin, Tetrahedron. 41 (1985) 3147-3152.

[22] K. Voiges, R. Adden, M. Rinken, P. Mischnick, Critical re-investigation of the alditol acetate method for analysis of substituent distribution in methyl cellulose, Cellulose 19 (2012) 993-1004.

[23] I. Unterieser, P. Mischnick, Labeling of oligosaccharides for quantitative mass spectrometry, Carbohydr. Res. 346 (2011) 68-75.

[24] S. L. R Ellison, A. Williams, Quantifying Uncertainty in Analytical Measurement, QUAM:2012.P1.

[25] Evaluation of measurement-Guide to the expression of uncertainty in measurement, JCGM 100:2008

[26] M. Wilm, Principles of electrospray ionization, Mol. Cell. Proteomics. 10 (2011) M111.009407.

[27] L. Tang, P. Kebarle, Dependence of ion intensity in electrospray mass spectrometry on the concentration of the analytes in the electrosprayed solution, Anal.Chem. 65 (1993) 3654-3668.

[28] P. Kebarle, U. H. Verkerk, Electrospray: from ions in solution to ions in the gas phase, what we know now, Mass Spectrom. Rev. 28 (2009) 898-917.

[29] R. Adden, A. Bösch, P. Mischnick, Novel possibilities by cationic ring-opening polymerisation of cyclodextrin derivatives: Preparation of a copolymer bearing block-like sequences of tri-Omethylglucosyl units, Macromol. Chem. Phys. 205 (2004) 2072-2079.

[30] I. Unterieser, J. Cuers, K. Voiges, J. Enebro, P. Mischnick, Quantitative aspects in electrospray ionization ion trap and matrix-assisted laser desorption/ionization time-of-flight mass spectrometry of malto-oligosaccharides, Rapid Commun. Mass Spectrom. 25 (2011) 2201-2208.

[31] C. J. Hogan Jr, P. Biswas, Monte carlo simulation of macromolecular ionization by nanoelectrospray, J. Am. Soc. Mass Spectrom. 19 (2008) 1098-1107.

[32] M. Karas, U. Bahr, T. Dülcks, Nano-electrospray ionization mass spectrometry: addressing analytical problems beyond routine, Fresenius. J. Anal. Chem. 366 (2000) 669-676.

[33]N. B. Cech, C. G. Enke, Practical implications of some recent studies in electrospray ionization fundamentals, Mass Spectrom. Rev. 20 (2002) 362-387. 